Cahiers $d u$ MONDE RUSSE

\section{Cahiers du monde russe}

Russie - Empire russe - Union soviétique et États indépendants

$43 / 4 \mid 2002$

Intellectuels et intelligentsia

\title{
S. Bertolissi, A. N. Saharov, eds, Konstitucionnye proekty v Rossii XVIII-načalo XX V.
}

\section{François-Xavier Coquin}

\section{OpenEdition}

\section{Journals}

\section{Édition électronique}

URL : https://journals.openedition.org/monderusse/4066

DOI : $10.4000 /$ monderusse. 4066

ISSN : $1777-5388$

\section{Éditeur}

Éditions de l'EHESS

Édition imprimée

Date de publication : 30 décembre 2002

ISBN : 2-7132-1796-2

ISSN : $1252-6576$

\section{Référence électronique}

François-Xavier Coquin, « S. Bertolissi, A. N. Saharov, eds, Konstitucionnye proekty v Rossii xvIIInačalo xx v. », Cahiers du monde russe [En ligne], 43/4 | 2002, mis en ligne le 18 juin 2009, consulté le 03 septembre 2022. URL : http://journals.openedition.org/monderusse/4066 ; DOI : https://doi.org/ 10.4000/monderusse.4066

Ce document a été généré automatiquement le 3 septembre 2022

Tous droits réservés 


\title{
S. Bertolissi, A. N. Saharov, eds, Konstitucionnye proekty v Rossii XVIII-načalo XX V.
}

\author{
François-Xavier Coquin
}

\section{RÉFÉRENCE}

\section{S. BERTOLISSI, A. N. SAHAROV, eds, Konstitucionnye proekty v Rossii XVIII-načalo} XX v. (Les projets constitutionnels en Russie, XVIII ${ }^{\mathrm{e}}$-début $\mathrm{XX}^{\mathrm{e}}$ siècle), Moscou, Institut Rossijskoj Istorii, RAN, 2000, 816 p.

1 C'est une lacune flagrante que cherche à combler ce recueil de projets constitutionnels russes publié par l'Institut d'histoire de l'Académie des sciences. Certes, le lecteur disposait bien pour cette même période de l'ouvrage déjà ancien de Marc Raeff ${ }^{1}$. Mais, quels que soient ses mérites, cet ouvrage qui réunissait neuf projets de réforme ne saurait de toute évidence rivaliser avec cet ambitieux recueil qui ne rassemble pas moins de cinquante projets constitutionnels couvrant les deux derniers siècles, ou peu s'en faut, de l'autocratie tsariste.

2 Même si ces projets leur étaient déjà plus ou moins familiers, les historiens éprouvaient toutefois quelque difficulté à consulter les originaux dispersés dans des publications savantes, d'accès parfois malaisé. Saluons donc, comme elle le mérite, cette publication qui met à la portée de tous, en langue originale (à l'occasion française), les projets constitutionnels les plus marquants de la période.

3 À cela ne se bornent pas les mérites des auteurs qui font précéder ce recueil de trois préfaces nourries qui retracent les avatars et le cheminement de l'idée constitutionnelle en Russie. Auteur d'un ouvrage récent sur Alexandre $\mathrm{I}^{\mathrm{er}}$ où figurent en bonne place les divers projets de "constitutionnalisme gouvernemental " élaborés à l'initiative de ce souverain $^{2}$, A. N. Saharov ouvre le feu et nous propose (p. 9-78) une relecture de l'histoire 
politique russe à la lumière de ces multiples projets successifs, restés, il est vrai, à l'état de projets.

4 Évoquant tout d'abord ce que l'on pourrait appeler leur préhistoire, l'auteur mentionne brièvement Ivan Kurbskij, dont la correspondance avec Ivan IV (dit le Terrible) peut être considérée comme le premier projet constitutionnel visant à limiter le pouvoir autocratique en associant l'aristocratie des bojare au gouvernement du pays. Mais Kurbskij ne parviendra pas à ouvrir une "alternative " à l'absolutisme autocratique, aussi peu que Boris Godunov ou le faux Dimitri qui n'aura guère le temps d'humaniser, ou mieux de "surmonter le despotisme " - pour reprendre l'expression de l'auteur (p. 22) - et d'engager ainsi la Russie sur une « voie de développement » différente.

Cela rappelé, « l'élan civilisateur » suivant interviendra peu après le règne de Pierre le Grand, indifférent, quant à lui, aux droits éventuels de ses sujets. L'avènement controversé en 1730 de sa nièce Anna Ivanovna, duchesse de Courlande, avait en effet incité quelques hauts dignitaires (les "verhovniki») à exiger de la nouvelle impératrice, préalablement à son accession au trône, le respect de certaines "conditions" qui prévoyaient une limitation du pouvoir autocratique au bénéfice, pour l'essentiel, de l'aristocratie titrée (doc. $n^{\circ} 1-3$ ), et que l'auteur nous invite à réévaluer. Sans aucunement contester le caractère aristocratique, déjà souligné par Raeff, desdites conditions, l'auteur juge que l'essentiel est ailleurs: non pas dans ce complot aristocratique, mais dans le fait que ce texte est, bien davantage, le premier, voire le seul, de tout le XVIII ${ }^{e}$ siècle à proposer, de manière indépendante du pouvoir, une limitation de l'autocratie et une transformation des structures politiques, et qu'il aurait ouvert, à ce titre, une première percée constitutionnelle.

6 Comme on le sait, le gros de la noblesse de service, dont les projets figurent également au recueil (doc. $\left.n^{\circ} 4-9\right)$, s'opposera à ces « conditions ", où les nobles de service voyaient un retour à l'ancienne domination des bojare, et ils permettront ainsi à Anna Ivanovna de reconduire le pouvoir autocratique illimité (doc. $\left.n^{\circ} 10\right)$. Bien que ces conditions aient, estime l'auteur (p. 40), « éveillé » le pays à l'idée constitutionnelle, l'opportunité offerte par la vacance du trône en 1730 ne sera pas saisie: la porte entrouverte par ce " prototype de projet constitutionnel » se refermera, et rien ne réfrénera l'arbitraire sanglant d'Anna Ivanovna et d'Élisabeth Petrovna.

7 L'idée constitutionnelle n'en survivra pas moins, et l'avènement de Catherine II ouvrira « une nouvelle boucle dans la spirale constitutionnelle» (p. 44). Dès l'année 1762, le comte N. I. Panin, précepteur du grand-duc Paul (le futur Paul Ir), présentait en effet un projet visant de nouveau à limiter le pouvoir impérial (doc. no 14 et 15) au profit notamment du Sénat et d'un "Conseil impérial », que Catherine II écartera. Désireuse toutefois (tout comme déjà ses prédécesseurs) de codifier les lois de l'empire pour mettre fin à l'arbitraire administratif, l'impératrice ne tardera pas à convoquer une "Commission du Code» pour laquelle elle rédigera son Instruction (ou Nakaz) bien connue, partiellement reproduite en version française originale (doc. $n^{\circ} 17$ ). Cette Grande Commission législative échouera, on le sait, à rédiger les lois fondamentales attendues ; mais le règne de l'impératrice, qui avait interdit de se désigner, dans les documents à son adresse, du nom d'esclave ( $\mathrm{rab}$ ) ou de serf (holop) de Sa Majesté Impériale, n'en marquera pas moins un "progrès stupéfiant" dans la vie politique, restée jusqu'alors " étonnamment somnolente et étonnamment cruelle », de la Russie (p. 47).

8 Sous l'effet des « vents civilisateurs » en provenance de l'Occident, le climat social avait changé au cours de son règne, comme en témoignent en 1783-1784 de nouveaux projets 
des frères Panin "sur les Lois fondamentales de l'État ", et un projet de manifeste d'avènement à l'usage du futur Paul $\mathrm{I}^{\text {er }}$ (doc. $\left.\mathrm{n}^{\circ} 18-20\right)$; et quinze ans plus tard, l'ancien conseiller de Catherine II aux affaires culturelles, Bezborodko, pourra présenter en 1799 un mémoire "sur les besoins de l'empire Russe " $\left(\right.$ doc. $\left.n^{\circ} 21\right)$ où il était le premier à placer chaque citoyen « sous l'égale protection des lois » (p. 306). Malgré la hardiesse de ce document à demi constitutionnel, Bezborodko n'encourra pas les foudres de l'empereur Paul $\mathrm{I}^{\text {er }}$. Tant le règne de Catherine II avait entretenu «la vitalité d'une alternative [constitutionnelle] à l'autocratie ».

À son tour, le règne d'Alexandre $\mathrm{I}^{\mathrm{er}}$ (1801-1825) coïncide avec une nouvelle « poussée des idées constitutionnelles»(p.50). L'initiateur en est cette fois l'empereur lui-même, "premier monarque russe libéral », dont le projet de "révolution légale » aurait pu mettre fin au pouvoir illimité, et donc corrupteur, de l'autocratie. Mais ses projets et ceux du Comité secret de ses «jeunes amis» (doc. $\left.\mathrm{n}^{\circ} 22\right)$ ne verront pas le jour: c'est ainsi que la "Charte octroyée au peuple» russe (1801) qui prévoyait, entre autres, de garantir la sécurité des biens et des personnes (doc. $\mathrm{n}^{\circ}$ 23), "restera à l'état d'alternative", c'est-à-dire de "possibilité historique avortée»(p.56), alors qu'elle semblait devoir inaugurer une « nouvelle ère » constitutionnelle.

Un même sort frappera également la plupart des projets de M. M. Speranskij (doc. $\mathrm{n}^{\circ}$ 24-26), à commencer par son «Introduction au code des lois » russes de 1809. Ce projet - le premier à formuler la nécessité d'une séparation des pouvoirs en Russie - échafaudait tout un édifice de doumas locales et régionales, couronné, on le sait, par une Douma d'État à compétences législatives, où siégeraient des représentants des différents ordres sociaux. Considéré par le préfacier comme un premier "sommet de la pensée constitutionnelle » en Russie (p. 58), ce projet aurait permis à la Russie de «progresser très rapidement [...] sur la voie d'un développement civilisé ", et de se transformer ainsi en une « monarchie constitutionnelle bourgeoise » et censitaire.

11 La disgrâce de Speranskij entraînera celle de ses projets de réforme; les traditions autocratiques l'emporteront, et Alexandre $\mathrm{I}^{\mathrm{er}}$, qui avait pourtant annoncé, dans son discours bien connu de mars 1818 devant la Diète de Pologne à Varsovie, son intention d'octroyer des institutions libérales (zakonno-svobodnye učrezdenija) à la Russie, ne tiendra pas parole. Certes, le projet de Charte constitutionnelle (ustavnaja gramota), élaboré à sa demande en 1820 par l'un de ses anciens «jeunes amis», N. N. Novosil'cev (doc. n²7), aurait pu à son tour replacer la Russie sur la voie de la monarchie constitutionnelle bourgeoise en garantissant l'égalité des citoyens devant la loi et en les dotant d'une large représentation nationale. Alexandre $\mathrm{I}^{\mathrm{er}}$ ' 'abstiendra toutefois de promulguer un texte qu'il avait pourtant approuvé. Aussi son règne sera-t-il finalement celui des occasions perdues ou des «alternatives avortées »; et la Russie continuera longtemps encore à se débattre dans le même cercle vicieux: la réforme de l'autocratie supposait en effet l'apparition d'une société capable d'appuyer les projets de réforme constitutionnelle, mais cette société civile ne pouvait émerger sans la réforme préalable de cette même autocratie.

L'enlisement de ces divers projets constitutionnels avait favorisé, vers la fin du règne d'Alexandre $\mathrm{I}^{\mathrm{er}}$, l'apparition de diverses sociétés secrètes à l'origine de l'insurrection du 14/26 décembre 1825. Paradoxalement, le projet constitutionnel du décabriste "libéral » N. M. Murav'ev (doc. $n^{\circ} 28$ ), inspiré en partie par les institutions américaines, concordait sur bien des points avec ce que l'auteur appelle le «constitutionnalisme larvé du gouvernement ». Aussi serait-il erroné, estime l'auteur, de ne voir avec Marc Raeff, dans les divers projets constitutionnels des décabristes, qu'un simple «complot 
aristocratique ». En réalité, les conjurés poursuivaient des buts beaucoup plus larges : non seulement combattre l'arbitraire autocratique et rétablir une "communication" entre le pouvoir et ses sujets, mais plus encore « faire passer délibérément la Russie d'un stade de civilisation à un autre", soit, précise l'auteur, d'un absolutisme féodal à une monarchie constitutionnelle moderne de type parlementaire (p.62). Et c'est l'ampleur même de leurs plans, auxquels ni le pays ni ses élites n'étaient aucunement préparés, qui serait avant tout, aux yeux de l'auteur, responsable de leur échec.

Quant à Pestel', dont laJustice russe (doc. $n^{\circ}$ 29) divisait la société entre "ceux qui commandent ", en raison de leur "supériorité morale », et "ceux qui obéissent », il jugeait nécessaire de commencer par « détruire la société préalablement à toute autre Action» (p. 478-479). C'était là tourner le dos à toute perspective constitutionnelle pour s'engager sur la voie d'une révolution radicale, et même extrémiste, qui devait conduire successivement à Nečaev, puis à la Volonté du peuple, et enfin à la terreur et à la dictature bolcheviques, en raison, nous dit l'auteur, du « retard culturel » de la Russie et de l'oppression subie par tout un peuple d'humiliés et d'offensés. Et ce fut le "péché historique » de l'autocratie que d'abandonner dès le début des années 1820 « l'initiative sociale » en raison de l'incapacité de la monarchie à réaliser des réformes en temps voulu (p. 62-63).

Quoi qu'il en soit, l'insurrection décabriste n'aura guère d'autres résultats que négatifs, et (tout comme naguère la révolte de Pugačev) elle interrompra la marche vers un régime constitutionnel qui aurait permis de reconstruire et de "civiliser» la Russie. Aussi faudra-t-il attendre le règne du tsar réformateur Alexandre II pour que le ministre de l'Intérieur P. A. Valuev élabore à son tour en 1863, au lendemain de l'insurrection polonaise, et à la demande (une fois de plus) du tsar, son projet bien connu de réforme du Conseil d'État (doc. $n^{\circ} 30$ et 31). Comme le rappelle l'auteur (p.65-66), ces projets recommandaient de « faire confiance au peuple » afin de lui permettre de prouver ainsi sa «maturité politique» face à une Pologne rebelle. Dans ce but, Valuev prévoyait d'associer le pays, par l'intermédiaire de ses représentants, à l'élaboration des lois, et de priver ainsi les « agitateurs révolutionnaires » d'une carte maîtresse. Mais, pour éviter de paraitre, à l'inverse, céder à leur pression, la réforme envisagée du Conseil d'État se ramenait à la simple présence de " conseillers " (glasnye) appelés à débattre, avec voix purement consultative, des seules questions pratiques qui leur seraient soumises.

Rien dans ce projet de " constitutionnalisme gouvernemental », sensiblement en retrait sur ceux de Speranskij ou de Novosil'cev, ne portait la moindre atteinte au monopole autocratique du pouvoir ; mais Alexandre II s'abstiendra néanmoins d'y donner suite. La «stagnation colossale» (p.67) de la pensée constitutionnelle se poursuivra donc; et il faudra attendre près de vingt ans pour que l'empereur charge en 1880 le général comte M. T. Loris-Melikov de réouvrir ce dossier afin d'apaiser le mécontentement de la "société » et de priver ainsi les terroristes de la Volonté du peuple du soutien de l'opinion. Persuadé que les mesures répressives ne pouvaient suffire, Loris-Melikov se bornera toutefois à préconiser une fois encore l'association (selon une procédure complexe) de représentants du pays à la seule discussion des lois; et ses plans destinés à " couronner » les grandes réformes des années 1860 manifestaient avant tout les limites de la pensée constitutionnelle officielle (doc. $n^{\circ}$ 33-39). Cette fois, il est vrai, Alexandre II devait approuver ce projet d'association d'élus à la discussion des lois ; et il s'apprêtait, on le sait, à le promulguer, lorsqu'il tombera ( $1^{\text {er }}$ mars 1881$)$ sous les bombes des "volontaires du peuple» qui stoppent net cette avancée constitutionnelle et qui 
rejettent ainsi la Russie plusieurs décennies en arrière, comme Loris-Melikov l'avait luimême prédit.

16 L'idée constitutionnelle est dès lors enterrée pour près de quinze ans (1881-1894). «L'européanisation » en cours de la Russie était encore trop superficielle, l'intelligentsia trop faible et la société trop peu diversifiée pour pouvoir contenir la contre-offensive des partisans de l'autocratie; et les successeurs d'Alexandre II n'auront pas la clairvoyance ni la lucidité voulues pour poursuivre dans la voie où l'empereur assassiné s'était engagé: celle d'un constitutionnalisme officiel décrété d'en haut. Corollaire inévitable, les milieux d'opposition en viennent progressivement à disputer l'initiative au souverain avec l'aide des forces sociales suscitées par les réformes des années 1860, et ils prennent la tête d'un mouvement constitutionnel par en bas, dirigé désormais contre le régime et qui se radicalise sous l'effet du raidissement autocratique.

On connaît la suite: les projets de constitution élaborés en pleine effervescence révolutionnaire par l'Union pour la libération et par le futur président de la première Douma, S. A. Muromcev (doc. $n^{\circ} 40-41$ ), puis le manifeste impérial mort-né du 6 août 1905 (doc. $n^{\circ} 42$ ) portant création d'une Douma d'État consultative, et enfin le « manifeste constitutionnel» du 17 octobre arraché à Nicolas II par la grève générale d'octobre 1905. Appliqué sans arrière-pensées, ce manifeste était de nature à engager enfin la Russie, avec un siècle de retard, sur la voie du développement constitutionnel et de la «normalité juridique». Tel ne sera pas le cas : Nicolas II s'ingéniera au contraire à restreindre au maximum ses promesses du 17 octobre et à faire passer la Douma nouveau-née sous la toise autocratique.

Simples manœuvres de retardement : comme l'avait exposé Witte dans son rapport au tsar du 18 août 1905 (doc. $\mathrm{n}^{\circ}$ 44), la société avait « dépassé le régime existant » (p. 745) qui ne voulait toujours entendre parler ni de constitution ni de suffrage « universel, égal, direct et secret ». Aussi la monarchie ne parviendra-t-elle plus à ressaisir l'initiative et à proposer un nouveau pacte gouvernemental au pays qui aspirait, selon Witte, à un véritable état de droit (pravovoj porjadok) sur la base des libertés civiques. Comme le prouvaient la dissolution coup sur coup des deux premières Doumas et la modification unilatérale des Lois fondamentales promulguées solennellement par le souverain en avril 1906, les droits octroyés au pays n'étaient guère que des droits en sursis, sans aucune garantie juridique autre que la bonne (ou la mauvaise) volonté impériale. Finalement la "monarchie du 3 juin» (1907) et Stolypin «interrompront le processus de démocratisation mis en route par le manifeste du 17 octobre qui n'était garanti par rien » (p. 76); et le fossé continuera donc à se creuser entre une autocratie sur le déclin et la société cultivée, ou encore "civilisée», car la (longue) marche vers un régime constitutionnel ne faisait qu'un, dans l'esprit du préfacier, avec le progrès même de la civilisation en Russie.

À ses yeux, l'État de droit, qui suppose un long apprentissage, n'avait toujours pas pris racine en Russie à la veille de la guerre, comme la révolution de 1917 en apportera la démonstration. Les meilleurs juristes de Russie auront beau élaborer, en prévision de l'Assemblée constituante, une loi électorale modèle et les meilleurs projets de régime intérimaire en attendant l'adoption de la constitution espérée, rien n'y fera. Ces projets resteront suspendus dans le vide, faute de tenir compte du niveau culturel et du niveau de conscience juridique du peuple russe qui n'était pas passé « par l'école séculaire de l'éducation civique et du développement constitutionnel» (p.78). Les conditions historiques nécessaires à leur réalisation faisaient tout simplement défaut. Aussi la 
révolution de 1917 suivra-t-elle inexorablement son cours, en l'absence des forces sociales capables d'en canaliser la progression; et le peuple russe ne voudra " rien savoir d'aucun gouvernement de droit et de constitution $»^{3}$. Étrangères aux traditions historiques de la Russie, les notions d'État de droit, de légalité et de régime constitutionnel ne parviendront pas réellement à prendre pied ; et ce que l'auteur appelle «le martyrologe politique de l'histoire russe» (p. 28) repartira de plus belle au grand dam de la société civile, des droits de l'homme et autres « chimères constitutionnelles ».

C'est cette même histoire que nous retrace, dans une optique plus nettement comparative et juridique, A. N. Meduševskij, auteur de différentes études sur l'autocratie et l'idée constitutionnelle. Sa préface (p. 95-166) prend la forme d'un petit traité sur l'histoire du constitutionnalisme russe, nourri d'emprunts étrangers, comme en font foi les « conditions » ou « articles » de 1730 qui ouvrent la liste des documents sélectionnés. Inspirées de la constitution suédoise de 1720, ces conditions visaient à limiter l'arbitraire personnel du souverain au profit de l'oligarchie titrée, dans l'esprit de la Moscovie d'avant Pierre le Grand. Mais ce « constitutionnalisme oligarchique » se heurtera, on l'a vu, à l'hostilité du gros de la noblesse provinciale qui lui oppose une petite poignée de contre-projets apparentés entre eux et destinés à refréner les ambitions de la haute noblesse de cour. Reproduits dans leur ordre chronologique (doc.4-9), ces projets reflétaient tout à la fois, souligne le préfacier, la conscience et la culture politiques de leurs auteurs, bien informés des institutions étrangères par l'intermédiaire des diplomates russes en poste à l'étranger, et notamment en Suède.

21 À son tour, l'avènement mouvementé de Catherine II avait suscité une deuxième vague de projets aristocratiques - ceux des frères Šuvalov, de Voroncov et de Panin (doc. 14-16) - visant derechef à renforcer le contrôle de la haute noblesse sur les actes du souverain, afin d'en limiter l'arbitraire tout particulièrement à l'honneur sous le règne d'Elizabeth Petrovna (1741-1761). Admiratrice de Montesquieu et plus ou moins sincèrement acquise aux Lumières, Catherine II, dont le Nakaz marque, selon le préfacier, "l'apogée de ces tentatives» de réformes (p.109), saura neutraliser cette contreoffensive nobiliaire grâce à sa Commission du Code de 1767-1768, bientôt interrompue par la guerre russo-turque (1768-1774). Apparemment infructueuse, cette Commission législative n'en illustrait pas moins le souci persistant qu'aura l'impératrice de faire prévaloir la " légalité » (zakonnost') dans le cadre d'une autocratie réglée et éclairée, bien distincte à ses yeux du despotisme, tel que le définit Montesquieu. Et l'intérêt de Catherine II pour le juriste anglais Blackstone, qui inspira notamment (on le sait) sa réforme des provinces de 1775, s'explique, nous rappelle l'auteur, fidèle à sa méthode comparative, par le fait que le théoricien britannique interprétait la séparation des pouvoirs au bénéfice du monarque, garant de la stabilité des institutions (p. 110).

Passons sur les projets déjà évoqués des frères Panin, et celui du conseiller Bezborodko «sur les besoins de l'empire russe». Quoique dévoué à l'autocratie, qu'une grande majorité de Russes jugeait seule adaptée à l'immensité d'un empire multi-national, ce dernier ne s'en prononçait pas moins, en bon disciple de Montesquieu et des Lumières, en faveur d'une monarchie réglée, encadrée par des lois fondamentales, qui s'imposeraient au souverain et en limiteraient l'arbitraire personnel (doc. $\left.n^{\circ} 21\right)$. À ce titre, le chancelier Bezborodko forme, aux yeux du préfacier, la transition entre le « Siècle des lumières » et le $\mathrm{XIX}^{\mathrm{e}}$ siècle, marqué par l'avènement d'Alexandre $\mathrm{I}^{\mathrm{er}}$ et l'apparition, dans le sillage de la Révolution française, de la "question constitutionnelle», qui commence à devenir un critère de légitimité politique en Europe. 

même "républicain", cherchera tout au long de son règne à réformer l'autocratie et à jeter les bases d'un "constitutionnalisme gouvernemental». Mais ni le projet de «Charte octroyée au peuple» russe (1801), dont le préfacier évoque au passage les sources occidentales, ni davantage les projets de Speranskij et ses plans de réforme de l'État ne verront le jour. Un même sort frappera également un ultime projet de Novosil 'cev (1820) préparé à la demande et sous le contrôle d'Alexandre $\mathrm{I}^{\mathrm{er}}$, mais dans le secret et à l'insu de la société. "Gouvernemental » ou " oligarchique », le constitutionnalisme russe butait en réalité sur la difficulté qu'il y avait à combiner le pouvoir illimité de l'autocratie avec une représentation nationale. Aussi restait-il essentiellement " fictif " et voué à faire longtemps encore figure de plante de serre, mal adaptée aux rigueurs du climat autocratique (p. 119).

C'est précisément cette incapacité de l'autocratie à se réformer de l'intérieur et à s'autolimiter qui devait engendrer les premières sociétés secrètes d'où sortiront les projets des décabristes: le «projet constitutionnel » de N. M. Murav'ev, inspiré en partie par les institutions américaines, et la Justice russe du colonel de la Garde impériale P. I. Pestel', tous deux reproduits in extenso dans le recueil (doc. 28 et 29). Mais pourquoi ne pas avoir également cité le manifeste (en date du 13 décembre 1825) du "dictateur» S.P. Trubeckoj, qui fait clairement ressortir les illusions et la dérive autoritaire du libéralisme nobiliaire?

Avec ces projets mieux connus que les précédents, d'accès parfois malaisé, l'auteur aborde un domaine plus familier à la plupart de ses lecteurs, notamment occidentaux. Ces derniers apprécieront son analyse des sources, jacobines notamment, mais également bonapartistes, de la Justice russe de Pestel', dont il souligne les différences avec la constitution de Murav'ev, leader de la Société du Nord (p. 123-124). Soucieux de ménager les transitions d'un régime autoritaire vers plus de démocratie, ce dernier était partisan, quant à lui, d'une monarchie constitutionnelle décentralisée et prévoyait même dans ce but de transférer la capitale de la Russie à Nijni-Novgorod (p.460). Au total, cette conjuration bicéphale inaugurait à sa façon, confirme le préfacier à la suite de Franco Venturi, les deux tendances antagonistes du mouvement russe de libération: libérale d'un côté et « autoritaro-révolutionnaire » de l'autre, avec Pestel', qui conduisait à ce que le préfacier appelle «l'impasse d'un régime autoritaire quasi [ou mieux: pseudo] constitutionnel » (p. 125).

Écrasée à contrecœur par Nicolas I ${ }^{\mathrm{er}}$, peu désireux d'inaugurer son règne par une effusion de sang, l'insurrection, et la répression qui suivra, frapperont d'interdit les idées constitutionnelles et libérales tout au long de son règne; et elles ne referont surface qu'avec les "grandes réformes » d'Alexandre II, pour lequel son ministre de l'Intérieur P. A. Valuev élaborera à sa demande, en 1863, un projet bien connu de réforme (limitée) du Conseil d'État (doc. 31), resté sans suite. Aussi faudra-t-il attendre la vague d'attentats organisés par la Volonté du peuple pour voir Alexandre II renouer avec sa politique réformatrice et faire appel, en février 1880 (cf. supra) à M. T. Loris-Melikov afin de combattre la « subversion ».

27 Conscient que les mesures répressives adoptées en réponse au premier attentat contre le tsar (1866), et aux suivants, ne pouvaient suffire, Loris-Melikov présentera entre avril 1880 et février 1881 et, à la demande du souverain, une série de rapports aisément consultables désormais (p. 628-674). Improprement appelés parfois « la constitution de Loris-Melikov », ces nouveaux projets de « constitutionnalisme gouvernemental », qui 
ne portaient aucunement atteinte au monopole politique de l'autocratie, avaient surtout le mérite de réouvrir le dossier des réformes et de faire un premier pas vers une constitution, comme l'avait expressément admis Alexandre II. Dans ce but, l'empereur avait fait préparer - à défaut de l'acte constitutionnel attendu par l'opinion pour le 19 février - du moins une déclaration gouvernementale dans ce sens (p. 658-663) en date du $1^{\mathrm{er}}$ mars $1881 \ldots$

«Grâce à Dieu, ce premier pas criminel et précipité vers une constitution n'a pas été accompli, et tout ce projet fantastique a été repoussé en conseil des ministres par une insignifiante minorité » (sic), annotera peu après Alexandre III sur le projet de LorisMelikov (p.640); et six mois ne se seront pas écoulés qu'Alexandre III promulguera le fameux "règlement provisoire" du 14 août 1881 qui méritera d'être appelé la constitution non écrite de la Russie. Les événements suivront donc un autre cours qui finira par aboutir à la révolution de 1905-1906, pour laquelle le recueil présente, nous l'avons vu, les deux projets d'inspiration libérale du mouvement constitutionnel : le projet de «Lois fondamentales» élaboré pour le mouvement de libération par «les meilleurs juristes russes", de tendance " résolument occidentaliste », qui ne croyaient pas - à la différence des samobytniki (ou partisans de la spécificité russe) - en la " possibilité pour le peuple russe de développer toutes les formes de liberté dans le cadre de l'absolutisme politique» (p. 146). Et, deuxième projet, celui du futur président de la première Douma, S. A. Muromcev, qui visait à assurer pacifiquement et par des voies légales le passage progressif vers une monarchie constitutionnelle, en faisant de la Douma l'acteur principal de la réforme politique et du contrôle du pays sur le pouvoir autocratique (p. 149).

Ces deux projets se verront bientôt concurrencés par le programme du parti cadet (ou constitutionnel-démocrate) mis au point lors du congrès fondateur de ce parti (12-18 octobre 1905). Pour l'essentiel, ce programme précisait les mécanismes juridiques et institutionnels destinés à assurer l'exercice de la souveraineté nationale attendue et à jeter les bases du futur État de droit : monarchie constitutionnelle ou république, entre lesquelles les cadets laissaient à l'Assemblée constituante à venir le soin de trancher. On ne pouvait imaginer contraste plus frappant qu'entre le savant équilibre de ce programme, longuement analysé par le préfacier (p.150-156), et celui des partis révolutionnaires peu soucieux, quant à eux, de constitution et des droits fondamentaux des citoyens.

30 Ainsi mis en appétit, le lecteur regrette seulement que ce projet constitutionnel fondé sur la distinction des pouvoirs et la décentralisation ait été, vu son ampleur, écarté du recueil, au profit de deux autres textes : le manifeste impérial du 6 août 1905, portant création d'une Douma consultative, et la Loi électorale concomitante (doc. $n^{\circ} 42-43$ ). Apparenté au projet d'assemblée consultative de Novosil'cev (1820), ce manifeste du 6 août (p. 716-728) qui rappelait l'époque d'Alexandre I ${ }^{\text {er }}$, manifestait le retard de la pensée officielle et faisait déjà figure, à peine publié, d'« anachronisme historique » (p. 74).

31 Enfin le recueil s'achève sur les divers projets élaborés par les constitutionnelsdémocrates entre mars et octobre 1917 (doc. 45-47). Mis au point dans le cadre de la Commission spéciale chargée de préparer la loi électorale en vue de la future Assemblée constituante, ces projets tendaient à faire enfin de la Russie un État de droit et représentaient l'aboutissement d'un siècle de réflexion et de travail constitutionnels. Qui mieux est, tout en ayant conscience que le gouvernement 
provisoire devrait passer la main dès la réunion de ladite Constituante et n'était donc nullement fondé à en régler le fonctionnement, les députés cadets avaient notamment tenu à ébaucher à son intention un modèle de système constitutionnel dont le préfacier analyse le détail avec précision et pertinence (p.149-156). En date du 20 octobre 1917 (!), ces projets (doc. 48-50), qui prévoyaient le fonctionnement du gouvernement intérimaire de transition entre la réunion de la Constituante et le vote de la future constitution, visaient notamment à prévenir toute dictature d'une assemblée unique de type jacobin... Le coup d'État du 25 octobre viendra balayer ce savant édifice; et il faudra attendre soixante-quinze ans, précise l'auteur, pour que la Russie renoue enfin avec cette "tradition juridico-constitutionnelle» brutalement interrompue, et restée jusque-là une pure construction intellectuelle sans être jamais parvenue à s'inscrire dans les faits.

Passons plus rapidement sur la contribution, plus limitée, de S.Bertolissi (p. 79-94): professeur à l'université de Naples, cet auteur s'interroge sur les raisons pour lesquelles ces projets constitutionnels qui tendaient à limiter le pouvoir souverain restèrent pratiquement sans suite - à la différence de l'Occident où l'évolution constitutionnelle ne cessa d'épouser (non sans retard parfois) l'évolution socio-économique de ces pays.

La principale cause de ces échecs répétés n'est autre, à ses yeux, que le servage qui s'étendait à l'ensemble de la population, "esclave " - selon Speranskij - soit du souverain, soit des pomeščiki (ou propriétaires fonciers nobles). De sorte que chez nous, concluait ce réformateur, institutions, distinctions sociales ou libertés, tout n'est qu'apparence ou fiction, à la merci de l'arbitraire autocratique (p. 87). À son tour, cette servitude généralisée ne permettait guère au peuple russe d'acquérir ne serait-ce qu'un minimum de conscience ou d'éducation politique ; et, d'Alexandre $\mathrm{I}^{\text {er }}$ à Nicolas II, les empereurs invoqueront régulièrement ce défaut de " maturité » pour refuser comme "prématurée " toute concession constitutionnelle; et le régime autocratique de continuer à osciller entre attraction occidentale et attachement aux traditions nationales.

Autre cause, liée à la précédente: le cloisonnement de la société en ordres, pourvus chacun de ses obligations et privilèges propres, faisait obstacle à la formation d'une société civile fondée sur l'égalité de droits et apte à contester le pouvoir du tsar qui apparaissait dès lors comme le seul garant de l'intérêt général. En raison de la persistance des ordres sociaux, même après les " grandes réformes » des années 1860, la bourgeoisie russe ne parviendra pas à jouer le rôle de porte-parole de l'idée constitutionnelle et se laissera cantonner, de l'aveu général, dans son culte du Veau d'or sans réellement travailler à l'émancipation, en panne, de la société. Et sa faiblesse interdira à cette bourgeoisie-croupion de prendre la tête du mouvement constitutionnel et libéral qui restera jusque vers 1905 l'apanage avant tout de la fraction éclairée de la noblesse.

À son tour, ce cloisonnement de la société en ordres faisait obstacle (souligne l'auteur) à la formation d'un État de droit auquel l'arbitraire autocratique n'avait nullement préparé les esprits. De fait, en l'absence d'un cadre juridique légal valable pour tous, les notions mêmes de droit et de loi, ou de légalité, variaient d'un ordre à l'autre. C'est ainsi que le monde paysan, maintenu en marge de la société dans un statut de seconde zone, avait un sentiment du droit et de la justice bien différent de celui des élites; et la conception de la légalité qui était celle du pouvoir n'avait rien de commun avec celle, moralisante et subversive, de toute une fraction de l'intelligentsia, comme l'auteur le rappelle à la suite de M. Raeff (p.90-91). En l'absence d'une culture juridique et de 
références communes, ces projets constitutionnels successifs, au demeurant secrets (censure oblige !) n'avaient guère de chances de s'imposer.

Élargissons le débat: pour faire prévaloir leurs vues, les auteurs de ces projets constitutionnels auraient dû, ajouterai-je, forcer la main au pouvoir; or personne, ou presque, n'y songeait. Largement dominant jusqu'en 1905, l'attachement à l'autocratie constituait même un obstacle quasi insurmontable. Comme l'avait écrit Bezborodko dans son mémoire de 1799, seule l'autocratie paraissait capable de maintenir, vu son immensité, la cohésion de l'empire russe, et « le plus minime affaiblissement du pouvoir autocratique aurait pour conséquence la perte (ottorÂenie) de nombreuses provinces, l'affaiblissement de l'État et des malheurs sans nombre pour la population » (p. 305). Cela restait vrai un siècle plus tard, et le réformateur Loris-Melikov, tout occidentaliste qu'il fût, déclarait lui-même qu'il préférerait se tuer plutôt que de limiter le pouvoir autocratique (p. 143).

Un tel propos n'était pas isolé: longtemps efficace, le régime autocratique formait un système tutélaire et cohérent, à l'ébranlement duquel tous avaient quelque chose à perdre, à commencer par les auteurs de ces divers " projets constitutionnels ». Serviteurs du souverain ou étrangers à la hiérarchie officielle et opposants en puissance, ils appartenaient tous, précisons-le, aux milieux dirigeants ou privilégiés et dépendaient à un titre ou à un autre de l'autocratie, qui constituait le principal facteur de stabilité en temps de crise. Aussi ne demandaient-ils guère qu'une représentation consultative, censitaire et par ordres, chargée de conseiller et d'assister le monarque; car une constitution de plein droit aurait signifié la fin de l'autocratie. Mais, à ne vouloir limiter l'autocratie qu'avec son consentement, les auteurs de ces projets se condamnaient à l'impuissance.

Enfin, on ne saurait davantage oublier que l'empereur était également le tsar orthodoxe, dont la foi était partagée par l'immense majorité du peuple russe, formé à voir dans son souverain l'instrument de la Providence divine, responsable à ce titre de la grandeur et de la prospérité du pays. Toute évolution constitutionnelle supposait donc une désacralisation du souverain et une laïcisation préalable de la société qui ne figuraient nullement, de tout le XIX ${ }^{e}$ siècle, à l'ordre du jour, et dont on peut regretter que les auteurs n'aient pas fait explicitement état. Lorsque Nicolas II avait refusé en décembre 1904, malgré l'avis de certains de ses conseillers, de mettre le doigt dans l'engrenage constitutionnel et de laisser ainsi la Russie "retomber dans le péché et la barbarie ", il avait clairement situé le débat: une réforme constitutionnelle irait à l'encontre des traditions morales, religieuses et nationales auxquelles le pays restait encore à cette date profondément attaché et auxquelles le tsar n'avait pas le pouvoir de renoncer.

Destinée à combattre toute contagion révolutionnaire, la devise «orthodoxie, autocratie, esprit national », formulée par Uvarov (1833) dans le sillage de la Guerre patriotique de 1812-1813, reflétait encore à cette date le fond de l'expérience historique du pays et un sentiment patriotique encore largement répandu. Anachronique ou non, ce credo politico-religieux faisait obstacle à toute percée constitutionnelle qui supposait que le tsar orthodoxe s'efface devant la personne de l'empereur, seul à pouvoir se muer en souverain constitutionnel. Désacraliser et "tuer", symboliquement ou non, le tsar constituait donc le préalable à toute évolution constitutionnelle et pouvait seul permettre de débloquer la situation.

Interrompons ici l'énumération des causes multiples de ce "martyrologe politique » à quoi A. N. Saharov assimile (p. 28) cette marche contrariée vers le constitutionnalisme, venu périodiquement frapper à la porte de la Russie. Évoquées, chemin faisant, par les 
auteurs, les raisons de ces échecs répétés mériteraient à elles seules une analyse approfondie que les éditeurs auront à cœur, espérons-le, de nous offrir un jour. Cette attente n'enlève rien, toutefois, aux mérites du présent recueil dont on ne saurait trop souligner l'intérêt et la richesse documentaire. Sans oublier les «notes et commentaires» en fin de volume (p. 779-812) qui apportent un éclairage historique bienvenu à la plupart des documents reproduits.

Remercions donc les éditeurs pour ce précieux ouvrage de référence qui réintègre ces tentatives constitutionnelles dans la trame de l'histoire russe et que tout historien de la Russie tsariste ne manquera pas de garder à portée de main. Même si l'on ne partage pas toujours l'optique résolument "occidentaliste » de ses auteurs, cet ouvrage témoigne à sa façon de l'ampleur prise ces derniers temps par le renouvellement de l'historiographie russe, et il constitue la contribution des auteurs au processus de réévaluation en cours. On se doit de leur en donner acte.

\section{NOTES}

1. Marc Raeff, Plans for political reform in Imperial Russia, 1730-1905, Englewood Cliffs, Prentice-Hall Inc., N.J., 1966, 159 p.

2. A. N. Saharov, Aleksandr I, Moscou, Nauka, 1998, 287 p.

3. Cf. Nicolas Berdiaeff, Un nouveau Moyen Âge, Paris, Plon, 1927, p. 176. 